\title{
LOCAL REGULARITY CONDITIONS ON INITIAL DATA FOR LOCAL ENERGY SOLUTIONS OF THE NAVIER-STOKES EQUATIONS
}

\author{
KYUNGKEUN KANG, HIDEYUKI MIURA, AND TAI-PENG TSAI
}

Dedicated to Hideo Kozono on the occasion of his 60th birthday

\begin{abstract}
We study the regular sets of local energy solutions to the Navier-Stokes equations in terms of conditions on the initial data. It is shown that if a weighted $L^{2}$ norm of the initial data is finite, then all local energy solutions are regular in a region confined by space-time hypersurfaces determined by the weight. This result refines and generalizes Theorems C and D of Caffarelli, Kohn and Nirenberg (Comm. Pure Appl. Math. 35; 1982) and our recent paper [15] as well.
\end{abstract}

\section{INTRODUCTION}

1.1. Regular sets for local energy solutions. We are concerned with the regularity of weak solutions of the incompressible Navier-Stokes equations

$$
\partial_{t} v-\Delta v+v \cdot \nabla v+\nabla p=0, \quad \operatorname{div} v=0
$$

in $\mathbb{R}^{3}$, associated with the initial value $\left.v\right|_{t=0}=v_{0}$ with $\operatorname{div} v_{0}=0$. The global existence of weak solutions for finite energy initial data was established by Leray [21], and extended to domains by Hopf [10]. Despite a lot of efforts since their foundational work, the global regularity of the weak solutions remains a longstanding open problem. It is known from works $[7,16,9]$ that for $v_{0} \in L^{q}\left(\mathbb{R}^{3}\right)$ with $q \geq 3$, (NS) has a regular solution defined on some short time interval where it satisfies the following estimate:

$$
\|v(t)\|_{L^{\infty}} \leq C\left(v_{0}\right) t^{-\frac{3}{2 q}}
$$

with the constant $C\left(v_{0}\right)=C_{q}\left\|v_{0}\right\|_{L^{q}}$. Motivated by the problem for constructing large forward self-similar solutions to (NS), Jia and Šverák [12] asked under which condition this result can be localized in space. For $B_{r}(x)=\left\{y \in \mathbb{R}^{3}:|x-y|<r\right\}$ and $B_{r}=B_{r}(0)$, their question can be stated as follows:

(R) If $v_{0}$ is a general initial data for which a suitable weak solution $v$ is defined and $\left.v_{0}\right|_{B_{2}} \in L^{q}\left(B_{2}\right)$, can we conclude that $v$ is regular in $B_{1} \times\left(0, t_{1}\right)$ for some time $t_{1}>0$ ?

2010 Mathematics Subject Classification. 35Q30, 76D05, 76D03.

Key words and phrases. Navier-Stokes equations, regular sets, local energy solutions.

We thank Professor Reinhard Farwig for valuable suggestions on Theorem 1.3. The research of Kang was partially supported by NRF-2019R1A2C1084685 and NRF-2015R1A5A1009350. The research of Miura was partially supported by JSPS grant 17K05312. The research of Tsai was partially supported by NSERC grant RGPIN-2018-04137. 
This question is settled affirmatively for the scale subcritical case $q>3$ in [12] and for the critical case $q=3$ in [1, 14]; see also [25] for a condition on the initial enstrophy and [1] for further extension to the $L^{3, \infty}$ space and the critical Besov spaces. In our previous work [15], we revisited this problem from a slightly different view point. Let us define the scaled local energy of the initial data by

$$
N_{(\alpha)}\left(v_{0}\right):=\sup _{r \in(0,1]} \frac{1}{r^{\alpha}} \int_{B_{r}}\left|v_{0}(x)\right|^{2} d x
$$

for $\alpha>0$. We showed that if $N_{(1)}$ (with $\alpha=1$ ) is sufficiently small, any local energy solution is regular and satisfies the estimate (1.1) in the region

$$
\left\{(x, t) \in B_{1} \times(0, \infty): c N_{(1)}^{2}|x|^{2} \leq t<t_{1}\right\}
$$

for some $t_{1}>0$; see Section 2 for the definition of the local energy solutions. Concerning the question $(\mathrm{R})$, the above result shows that if the local Morrey norm defined by

$$
\|f\|_{m^{2,1}\left(B_{2}\right)}:=\sup _{x_{0} \in B_{2}, r \in(0,1]}\left(\frac{1}{r} \int_{B_{r}\left(x_{0}\right) \cap B_{2}}|f(x)|^{2} d x\right)^{\frac{1}{2}}
$$

is sufficiently small, the local energy solution is regular in $B_{1} \times\left(0, t_{1}\right)$ for some $t_{1}>0$ and satisfies the $L^{\infty}$ bound (1.1). By the well-known relation $m^{2,1} \hookleftarrow L^{3}$, the latter recovers the results on the local regularity results $[1,14]$ for $L^{3}$ data. The goal of the present work is to study the local in space regularity of the solutions with data satisfying the condition $N_{(\alpha)}<\infty$ for general $\alpha>1$. Intuitively, the bigger $\alpha$ is, the more regular the data is, and so is the solution at least locally. We justify it in the following sense: The solution is regular in a larger region and it also satisfies an improved $L^{\infty}$ estimate in a slightly different region. More precisely, it can be stated as follows.

Theorem 1.1. Assume that a local energy solution $v$ in $\mathbb{R}^{3} \times(0, T)$ with the initial data $v_{0} \in L_{\text {uloc }}^{2}$ satisfies $N_{(\alpha)}<\infty$ for some $\alpha>1$. Then there exists $T_{1}=$ $T_{1}\left(\left\|v_{0}\right\|_{L_{\text {uloc }}^{2}}, N_{(\alpha)}, \alpha, T\right)>0$ such that $v$ is regular in the region

$$
\Pi_{1}=\left\{(x, t) \in B_{\frac{1}{2}} \times(0, T): c_{1} N_{(\alpha)}^{2}|x|^{2 \alpha} \leq t<T_{1}\right\}
$$

and satisfies

$$
|v(x, t)| \leq C t^{-\frac{1}{2}} \quad \text { for }(x, t) \in \Pi_{1} .
$$

Moreover, if $\alpha \in(1,4)$, there exist $T_{2}=T_{2}\left(\left\|v_{0}\right\|_{L_{u l o c}^{2}}, N_{(\alpha)}, \alpha, T\right)>0$ and $M=$ $M\left(\left\|v_{0}\right\|_{L_{u l o c}^{2}}, N_{(\alpha)}, \alpha\right)>0$ such that

$$
|v(x, t)| \leq M t^{\frac{\alpha-3}{4}} \quad \text { for }(x, t) \in \Pi_{2}
$$

holds with

$$
\Pi_{2}=\left\{(x, t) \in B_{\frac{1}{2}} \times(0, T): \frac{c_{2} N_{(\alpha)}^{\frac{2}{\alpha}}}{\left(1+N_{(\alpha)}\right)^{2+\frac{2}{\alpha}}}|x|^{2} \leq t<T_{2}\right\} .
$$

Here $c_{1}, c_{2}$, and $C$ are positive constants depending only on $\alpha$. 


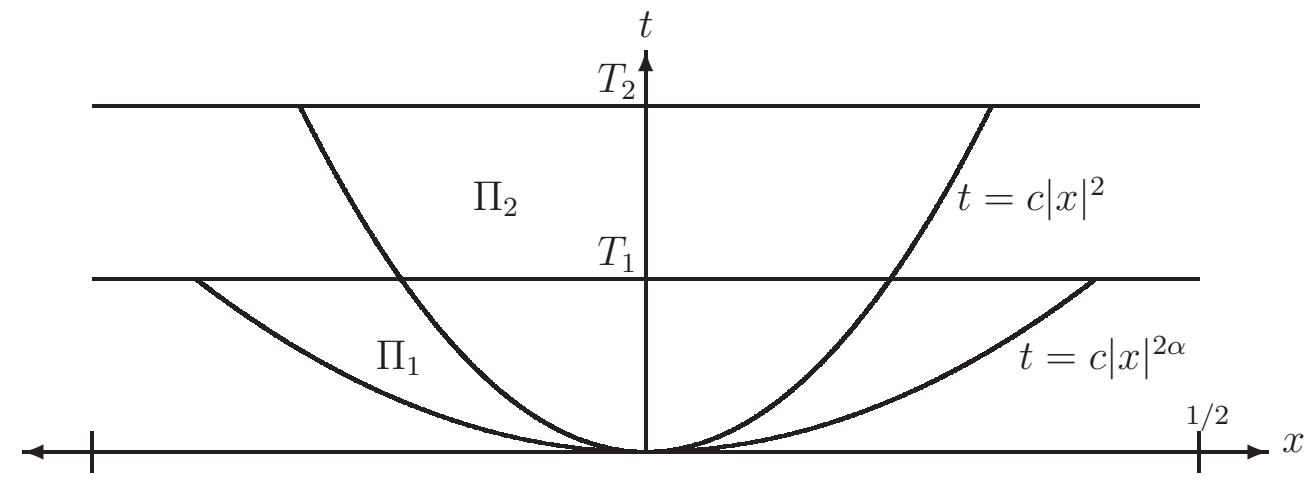

FiguRE 1. Regular regions for Theorem 1.1 for the case $T_{2}>T_{1}$.

Comments for Theorem 1.1:

a) Theorem 1.1 is also valid for $\alpha=1$ if we further assume smallness of $N_{(1)}$. In that case we recover Theorem 3.4 of [15].

b) In Theorem 3.1 we will give a more general statement for $\alpha \in(1,4)$ in terms of

$$
N_{(\alpha), R}:=\sup _{r \in(R, 1]} \frac{1}{r^{\alpha}} \int_{B_{r}(0)}\left|v_{0}\right|^{2} \quad\left(0 \leq R \leq \frac{1}{2}\right) .
$$

c) $T_{1}$ will be given by (3.30). $T_{2}$ will be given by (3.2) as $S_{2}$ with $N_{R}=N_{(\alpha), 0}$. None is necessarily larger than the other.

As one of applications of our result to some specific cases, we show a regularizing estimate of the form (1.1) for locally $L^{q}$ data, $3<q \leq \infty$.

Theorem 1.2. Let $(v, p)$ be a local energy solution in $\mathbb{R}^{3} \times(0, T)$ with the initial data $v_{0} \in L_{\mathrm{uloc}}^{2}$. If $v_{0} \in L^{q}\left(B_{2}\right)$ for some $q \in(3, \infty]$, then there exists $T_{3}=$ $T_{3}\left(q,\left\|v_{0}\right\|_{L^{q}\left(B_{2}\right)},\left\|v_{0}\right\|_{L_{\mathrm{uloc}}^{2}}\right)>0$ such that $v$ is regular in $B_{1} \times\left(0, T_{3}\right)$ and satisfies

$$
\|v(t)\|_{L^{\infty}\left(B_{1}\right)} \leq C\left(1+\left\|v_{0}\right\|_{L^{q}\left(B_{2}\right)}\right)^{3-\frac{6}{q}}\left\|v_{0}\right\|_{L^{q}\left(B_{2}\right)} t^{-\frac{3}{2 q}} \quad \text { for } t \in\left(0, T_{3}\right)
$$

with an absolute constant $C>0$.

Estimate (1.5) does not explicitly depend on $\left\|v_{0}\right\|_{L_{\text {uloc }}^{2}}$. Its dependence on $\left\|v_{0}\right\|_{L_{\text {uloc }}^{2}}$ is through the time upper bound $T_{3}$. In [2, Theorem 3], Barker and Prange obtained an $L^{\infty}$ bound in some time interval for locally $L^{6}$ initial data with the aim of the behavior of the $L^{3}$ norm near the singular point.

In [5], Caffarelli, Kohn, and Nirenberg established estimates of the regular sets for the suitable weak solutions for data in $L^{2}$ weighted spaces. Define weighted $L^{2}$ norm

$$
\left\|v_{0}\right\|_{L^{2, \beta}}:=\left\||x|^{\frac{\beta}{2}} v_{0}\right\|_{L^{2}\left(\mathbb{R}^{3}\right)}
$$

for $\beta \in \mathbb{R}$. It is shown in [5] that if the finite energy data satisfies $\left\|v_{0}\right\|_{L^{2,1}}<\infty$, then there exists a suitable weak solution which is regular in the set of points satisfying $t>\min \left(C, K|x|^{-2}\right)$. Moreover it is also shown that if the finite energy data satisfies $\left\|v_{0}\right\|_{L^{2,-1}}<\epsilon_{0}$ with some absolute small constant $\epsilon_{0}>0$, then there exists a suitable weak solution which is regular in the set $t>C|x|^{2}$; see [6] for its refinement. In [15, Corollary 1.4], we extended estimates of the regular sets for general data in $L^{2, \beta}$, 
$\beta \geq-1$. By using Theorem 1.1, we are able to show similar results for $\beta$ below -1 at least locally in time.

Theorem 1.3. Let $(v, p)$ be a local energy solution in $\mathbb{R}^{3} \times(0, T)$ for the initial data $v_{0} \in L_{\text {uloc }}^{2}$. Assume that $v_{0} \in L^{2, \beta}\left(\mathbb{R}^{3}\right)$ for some $\beta<-1$. Then there exist positive constants $T_{4}=T_{4}\left(v_{0}\right)$ and $c\left(v_{0}\right)$ such that $v$ is regular in the set

$$
\left\{(x, t) \in \mathbb{R}^{3} \times(0, T): c\left(v_{0}\right)|x|^{-2 \beta} \leq t<T_{4}\left(v_{0}\right)\right\} .
$$

The proofs of Theorems 1.1, 1.2 and 1.3 are based on a local-in-space a priori estimate for the scaled local energy defined by

$$
E_{r}(t):=\operatorname{ess~sup}_{0<s<t} \frac{1}{r^{\alpha}} \int_{B_{r}}|v(s)|^{2}+\frac{1}{r^{\alpha}} \int_{0}^{t} \int_{B_{r}}|\nabla v|^{2},
$$

which is useful to focus on the local regularity at the origin and is used in [15] for the case $\alpha=1$. In order to deal with the non-local effect of the pressure term, we apply the decomposition formula of $[19,17]$ to the local energy estimate. Our key observation is that the (global) $L_{\text {uloc }}^{2}$ norm of the initial data is sufficient to control the non-local effect at least locally in time; see Lemma 3.2. It should be noted that our strategy is different from previous works $[12,1,14]$ and that ours provides a rather direct and transparent approach.

1.2. Outline of the paper and notation. In section 2, we introduce the notion of local energy solutions and recall the local regularity criterion due to [5] as well as some technical lemmas. Section 3 is devoted to stating and proving our main results including Theorem 1.1.

Throughout this paper, $C \in(0, \infty)$ denotes an absolute constant which may change line by line.

\section{Preliminaries}

In this section, we recall some notions about the weak solution to (NS) and some results such as the $\epsilon$-regularity theorems and a priori estimates for the solutions.

For any domain $\Omega \subset \mathbb{R}^{3}$ and open interval $I \subset(0, \infty)$, we say $(v, p)$ is a suitable weak solution in $\Omega \times I$ if it satisfies (NS) in the sense of distributions in $\Omega \times I$,

$$
v \in L^{\infty}\left(I ; L^{2}(\Omega)\right) \cap L^{2}\left(I ; \dot{H}^{1}(\Omega)\right), \quad p \in L^{3 / 2}(\Omega \times I),
$$

and the local energy inequality:

$$
\begin{aligned}
& \int_{\Omega}|v(t)|^{2} \phi(t) d x+2 \int_{0}^{t} \int_{\Omega}|\nabla v|^{2} \phi d x d t \\
& \leq \int_{0}^{t} \int_{\Omega}|v|^{2}\left(\partial_{t} \phi+\Delta \phi\right) d x d t+\int_{0}^{t} \int_{\Omega}\left(|v|^{2}+2 p\right)(v \cdot \nabla \phi) d x d t
\end{aligned}
$$

for all non-negative $\phi \in C_{c}^{\infty}(\Omega \times I)$. Note that no boundary condition is required.

We next define the notion of local energy solutions, The following definition is formulated in [4], which is slightly revised from the notions of the local Leray solution defined in [19], the local energy solution in [17] and the Leray solution in [12]. We refer to [14, Section 2] for discussion of their relation. 
Definition 2.1 (Local energy solutions [4]). A vector field $v \in L_{\text {loc }}^{2}\left(\mathbb{R}^{3} \times[0, T)\right)$ is a local energy solution to (NS) with divergence free initial data $v_{0} \in L_{\text {uloc }}^{2}$ if

(1) for some $p \in L_{\text {loc }}^{3 / 2}\left(\mathbb{R}^{3} \times[0, T)\right)$, the pair $(v, p)$ is a distributional solution to $(\mathrm{NS})$,

(2) for any $R>0$,

$$
\operatorname{esssup}_{0 \leq t<\min \left\{R^{2}, T\right\}} \sup _{x_{0} \in \mathbb{R}^{3}} \int_{B_{R}\left(x_{0}\right)}|v|^{2} d x+\sup _{x_{0} \in \mathbb{R}^{3}} \int_{0}^{\min \left\{R^{2}, T\right\}} \int_{B_{R}\left(x_{0}\right)}|\nabla v|^{2} d x d t<\infty,
$$

(3) for all compact subsets $K$ of $\mathbb{R}^{3}$ we have $v(t) \rightarrow v_{0}$ in $L^{2}(K)$ as $t \rightarrow 0^{+}$,

(4) $(v, p)$ satisfies the local energy inequality (2.1) for all non-negative functions $\phi \in C_{c}^{\infty}(Q)$ with all cylinder $Q$ compactly supported in $\mathbb{R}^{3} \times(0, T)$,

(5) for every $x_{0} \in \mathbb{R}^{3}$ and $r>0$, there exists $c_{x_{0}, r} \in L^{3 / 2}(0, T)$ such that

$$
\begin{aligned}
p(x, t)-c_{x_{0}, r}(t) & =\frac{1}{3}|v(x, t)|^{2}+p \cdot v \cdot \int_{B_{3 r}\left(x_{0}\right)} K(x-y): v(y, t) \otimes v(y, t) d y \\
& +\int_{\mathbb{R}^{3} \backslash B_{3 r}\left(x_{0}\right)}\left(K(x-y)-K\left(x_{0}-y\right)\right): v(y, t) \otimes v(y, t) d y
\end{aligned}
$$

in $L^{3 / 2}\left(B_{2 r}\left(x_{0}\right) \times(0, T)\right)$, where $K(x)=\nabla^{2}\left(\frac{1}{4 \pi|x|}\right)$, and

(6) for any compact supported functions $w \in L^{2}\left(\mathbb{R}^{3}\right)^{3}$,

$$
\text { the function } t \mapsto \int_{\mathbb{R}^{3}} v(x, t) \cdot w(x) d x \quad \text { is continuous on }[0, T) \text {. }
$$

Let us also recall the uniformly local $L^{q}$ spaces for $1 \leq q<\infty$. We say $f \in L_{\mathrm{uloc}}^{q}$ if $f \in L_{\mathrm{loc}}^{q}\left(\mathbb{R}^{3}\right)$ and

$$
\|f\|_{L_{\mathrm{uloc}}^{q}}=\sup _{x \in \mathbb{R}^{3}}\|f\|_{L^{q}\left(B_{1}(x)\right)}<\infty .
$$

Local in time existence of local energy solutions for initial data in $L_{\text {uloc }}^{2}$ and also global existence for initial data in $E^{2}:=\bar{C}_{0}^{\infty} L_{\text {uloc }}^{2}$ and the weighted $L^{2}$ spaces are established in [19] and [8, 3], respectively. One of the advantage of the local energy solution is that it can be defined even for infinite energy data; see [18, 20, 13] and references therein for further developments and its applications, and [23] for the local energy solutions in the half space.

The next lemma shows a priori bounds for the local energy solution, where the crucial part is proved by Lemarié-Rieusset [19] and later revised in [17, 11]. The following version is deduced from $[14,4]$.

Lemma 2.2 (a priori bound of local energy solutions). Suppose $(v, p)$ is a local energy solution to (NS) defined in $\mathbb{R}^{3} \times(0, T)$ with divergence free initial data $v_{0} \in L_{\mathrm{uloc}}^{2}$. For 
any $s, q>1$ with $\frac{2}{s}+\frac{3}{q}=3$, there exist $C(s, q)>0$ and $c_{x_{0}}(t) \in \mathbb{R}^{3}$ such that

$$
\begin{aligned}
& E_{\text {uloc }}(t):=\operatorname{ess~sup~}_{0 \leq \tau \leq t} \| v(\tau)\left\|_{L_{\text {uloc }}^{2}}^{2}+\sup _{x_{0} \in \mathbb{R}^{3}} \int_{0}^{t} \int_{B_{1}\left(x_{0}\right)}|\nabla v|^{2} \leq 2\right\| v_{0} \|_{L_{\text {uloc }}^{2}}^{2} \\
& \sup _{x_{0} \in \mathbb{R}^{3}}\left\|p-c_{x_{0}}\right\|_{L^{s}\left(0, t ; L^{q}\left(B_{1}\left(x_{0}\right)\right)\right)} \leq C(s, q)\left\|v_{0}\right\|_{L_{\text {uloc }}^{2}}^{2}
\end{aligned}
$$

for $t \leq T_{\mathrm{uloc}}:=\min \left(T, \frac{c_{0}}{1+\left\|v_{0}\right\|_{L_{\mathrm{uloc}}^{4}}^{4}}\right)$ with a universal constant $c_{0}>0$. Similar estimates with $B_{1}$ replaced by $B_{r}$ are valid.

We now recall the scaled version of the $\epsilon$-regularity theorem of Caffarelli-KohnNirenberg [5, Proposition 1]. It is formulated in the present form in [24, 22].

Lemma 2.3. There are absolute constants $\epsilon_{C K N}$ and $C_{C K N}>0$ with the following property. Suppose that $(v, p)$ is a suitable weak solution of $(N S)$ in $B_{r}\left(x_{0}\right) \times\left(t_{0}-\right.$ $\left.\left.r^{2}, t_{0}\right)\right), r>0$, with

$$
\frac{1}{r^{2}} \int_{t_{0}-r^{2}}^{t_{0}} \int_{B_{r}\left(x_{0}\right)}|v|^{3}+|p|^{3 / 2} d x d t \leq \epsilon
$$

for some $\epsilon \leq \epsilon_{C K N}$, then $v \in L^{\infty}\left(B_{\frac{r}{2}\left(x_{0}\right)} \times\left(t_{0}-\frac{r^{2}}{4}, t_{0}\right)\right)$ and

$$
\|v\|_{L^{\infty}\left(B_{\frac{r}{2}\left(x_{0}\right)} \times\left(t_{0}-\frac{r^{2}}{4}, t_{0}\right)\right)} \leq \frac{C_{C K N} \epsilon^{\frac{1}{3}}}{r} .
$$

We recall a useful Gronwall-type inequality from [4, Lemma 2.2].

Lemma 2.4. Suppose $f \in L_{\mathrm{loc}}^{\infty}\left(\left[0, T_{0}\right) ;[0, \infty)\right.$ ) (which may be discontinuous) satisfies, for some $a, b>0$, and $m \geq 1$,

$$
f(t) \leq a+b \int_{0}^{t}\left(f(s)+f(s)^{m}\right) d s \quad \text { for } t \in\left(0, T_{0}\right),
$$

then we have $f(t) \leq 2 a$ for $t \in(0, T)$ with

$$
T=\min \left(T_{0}, \frac{C}{b\left(1+a^{m-1}\right)}\right)
$$

where $C$ is a universal constant.

Finally we also show the following elementary bound for the scaled energy.

Lemma 2.5. Assume that $f \in L_{\mathrm{loc}}^{2}\left(\mathbb{R}^{3}\right)$ and let $N_{R}=N_{(\alpha), R}(f):=\sup _{r \in(R, 1]} \frac{1}{r^{\alpha}} \int_{B_{r}}|f|^{2}<$ $\infty$ for some $\alpha>0$ and $R \in\left[0, \frac{1}{2}\right]$.

(i) If $\delta \geq 2^{\alpha} N_{R}$, then for any $x_{0} \in B_{\frac{1}{2}}$ we have

$$
\sup _{R\left(x_{0}\right)<r \leq 1-\left|x_{0}\right|} \frac{1}{r^{\alpha}} \int_{B_{r}\left(x_{0}\right)}|f|^{2} \leq \delta
$$

with $R\left(x_{0}\right)=\max \left(\frac{R}{2}, 2\left(\frac{N_{R}}{\delta}\right)^{\frac{1}{\alpha}}\left|x_{0}\right|\right)$. 
(ii) If $\alpha \geq 1$ and $\delta \geq 2 N_{R}$, then for any $x_{0} \in B_{\frac{1}{2}}$ we have

$$
\sup _{R^{\prime}\left(x_{0}\right)<r \leq 1-\left|x_{0}\right|} \frac{1}{r} \int_{B_{r}\left(x_{0}\right)}|f|^{2} \leq \delta
$$

with $R^{\prime}\left(x_{0}\right)=\max \left(\frac{R}{2}, \frac{2^{\alpha} N_{R}}{\delta}\left|x_{0}\right|^{\alpha}\right)$.

Proof. (i) Assume that $r \in\left(R\left(x_{0}\right), 1-\left|x_{0}\right|\right]$. If $1 / 2<r \leq 1-\left|x_{0}\right|$,

$$
\frac{1}{r^{\alpha}} \int_{B_{r}\left(x_{0}\right)}|f|^{2} \leq \frac{1}{r^{\alpha}} \int_{B_{1}(0)}|f|^{2} \leq \frac{1}{r^{\alpha}} N_{R} \leq \delta .
$$

If $\left|x_{0}\right| \leq r \leq 1 / 2,(2.9)$ clearly holds by the following estimate:

$$
\frac{1}{r^{\alpha}} \int_{B_{r}\left(x_{0}\right)}|f|^{2} \leq \frac{1}{r^{\alpha}} \int_{B_{2 r}(0)}|f|^{2} \leq 2^{\alpha} N_{R} \leq \delta
$$

where we have used $\left|x_{0}\right|+r \leq 2 r$. Finally, if $R\left(x_{0}\right)<r<\left|x_{0}\right|$ (this case is empty if $\left.\left|x_{0}\right| \leq R / 2 \leq R\left(x_{0}\right)\right)$, we have $\left|x_{0}\right|+r<2\left|x_{0}\right|$, and hence

$$
\frac{1}{r^{\alpha}} \int_{B_{r}\left(x_{0}\right)}|f|^{2} \leq \frac{1}{r^{\alpha}} \int_{B_{2\left|x_{0}\right|}(0)}|f|^{2} \leq\left(\frac{2\left|x_{0}\right|}{r}\right)^{\alpha} N_{R} .
$$

The right hand side is bounded by $\delta$ since $r>R\left(x_{0}\right) \geq 2\left(\frac{N_{R}}{\delta}\right)^{\frac{1}{\alpha}}\left|x_{0}\right|$. Therefore we have verified (2.9).

(ii) We note that if $R / 2<r<\left|x_{0}\right|$,

$$
\frac{1}{r} \int_{B_{r}\left(x_{0}\right)}|f|^{2} \leq \frac{1}{r} \int_{B_{2\left|x_{0}\right|}(0)}|f|^{2} \leq \frac{\left(2\left|x_{0}\right|\right)^{\alpha}}{r} N_{R} .
$$

Hence the right hand side is bounded by $\delta$ when $r \geq \frac{2^{\alpha} N_{R}}{\delta}\left|x_{0}\right|^{\alpha}$. The other case is similar to the proof of (i), and we omit the details.

Remark 2.6. In our previous paper [14], we have used the scale critical Herz norm in $\mathbb{R}^{3}$ :

$$
\left.\|f\|_{K_{p, \infty}^{-1+\frac{3}{p}}}:=\sup _{x_{0} \neq 0}\left|x_{0}\right|^{1-\frac{3}{p}}\|f\|_{L^{p}\left(B \frac{\left|x_{0}\right|}{2}\right.}\left(x_{0}\right)\right)
$$

for $p \geq 3$. We easily observe that

$$
\|f\|_{K_{2, \infty}^{\frac{1}{2}}}^{2} \leq C \sup _{r>0} \frac{1}{r} \int_{B_{r}}|f|^{2} d x \leq C^{\prime}\|f\|_{K_{2, \infty}^{\frac{1}{2}}}^{2}
$$

with some absolute constants $C$ and $C^{\prime}>0$.

\section{MAin Results}

In this section we prove our main results, Theorems 1.1-1.3, regarding the regularity of the local energy solutions. They are obtained as consequences of the following theorem.

Theorem 3.1. Let $v$ be a local energy solution of (NS) in $\mathbb{R}^{3} \times(0, T)$ associated with initial data $v_{0} \in L_{\text {uloc }}^{2}$, and

$$
N_{R}=N_{(\alpha), R}\left(v_{0}\right):=\sup _{r \in(R, 1]} \frac{1}{r^{\alpha}} \int_{B_{r}(0)}\left|v_{0}\right|^{2}<\infty
$$


for some $\alpha \in(1,4)$ and $R \in\left[0, \frac{1}{2}\right]$. There are absolute constants $c$ and $C>0$ such that the following holds.

(i) If $N_{R} \leq 1$ and $R \leq c \sqrt{S_{1}}$ with

$$
S_{1}:=\min \left(T, \frac{c}{1+\left\|v_{0}\right\|_{L_{\text {uloc }}^{2}}^{12}}\right)
$$

then we have

$|v(x, t)| \leq C\left[1+\left\|v_{0}\right\|_{L_{\text {uloc }}^{2}} S_{1}^{\frac{1}{4}(4-\alpha)}\right] t^{\frac{1}{4}(\alpha-3)} \quad$ for $\quad|x| \leq \frac{1}{2}, \quad c \max \left(R^{2}, N_{R}^{2 / \alpha}|x|^{2}\right) \leq t \leq S_{1}$.

(ii) If $N_{R} \geq 1$, assume $R \leq c N_{R} \sqrt{S_{2}}$ with

$$
S_{2}=\min \left(T, \frac{c}{1+\left\|v_{0}\right\|_{L_{\mathrm{uloc}}^{2}}^{12}},\left(\frac{c}{N_{R}}\right)^{\frac{2 \alpha+2}{\alpha-1}}, \frac{c}{\left(1+\left\|v_{0}\right\|_{L_{\mathrm{uloc}}^{2}}^{4 / 3}\right) N_{R}^{2}}\right),
$$

then we have

$$
\begin{array}{r}
|v(x, t)| \leq C\left[N_{R}^{(\alpha+1) / 2}+N_{R}^{3 / 2}\left\|v_{0}\right\|_{L_{\mathrm{uloc}}^{2}} S_{2}^{\frac{1}{4}(4-\alpha)}\right] t^{\frac{1}{4}(\alpha-3)} \\
\quad \text { for } \quad|x| \leq \frac{1}{2}, \quad \frac{c}{N_{R}^{2}} \max \left(R^{2},|x|^{2}\right) \leq t \leq S_{2} .
\end{array}
$$

The following local energy estimate is our key ingredient for the proof of Theorem 3.1, which shows the smallness of the scaled local energy $E_{r}$ defined in (1.7) is propagated at least locally in time and some scales.

Lemma 3.2. Let $v$ be a local energy solution of (NS) in $\mathbb{R}^{3} \times(0, T)$ associated with initial data $v_{0} \in L_{\text {uloc }}^{2}$, and $N_{R}=\sup _{R<r \leq 1} \frac{1}{r^{\alpha}} \int_{B_{r}(0)}\left|v_{0}\right|^{2}<\infty$ for some constants $\alpha \in[1,4)$ and $R \in[0,1)$. Let $\delta \geq 70 N_{R}$. Then for any $r \in(R, 1]$ we have

$$
E_{r}(t) \leq \delta \quad \text { for } 0<t \leq \min \left(\lambda r^{2}, S_{1}\right)
$$

where

$$
\lambda=\frac{c}{1+\delta^{2}} \leq 1 \quad \text { and } \quad S_{1}=\min \left(T, \frac{c \min \left\{1, \delta^{4}\right\}}{1+\left\|v_{0}\right\|_{L_{\mathrm{uloc}}^{2}}^{12}}\right) .
$$

Moreover, if $R<R_{1}:=\min \left(\sqrt{\frac{S_{1}}{\lambda}}, 1\right)$, there exists $c_{2}(t) \in \mathbb{R}$ such that

$$
\frac{1}{r^{2}} \int_{0}^{\lambda r^{2}} \int_{B_{r}}|v|^{3}+\left|p-c_{2}(t)\right|^{\frac{3}{2}} d x d t \leq C\left(\lambda^{\frac{1}{4}} \delta^{\frac{3}{2}} r^{\frac{3}{2}(\alpha-1)}+\lambda\left\|v_{0}\right\|_{L_{\mathrm{uloc}}^{2}}^{3} r^{\frac{9}{2}}\right) \quad \text { for all } r \in\left(R, R_{1}\right] .
$$

Above $c$ and $C>0$ are absolute constants.

Proof of Lemma 3.2. By the definition of the local energy solution, for $r \in(R, 1]$, there exists $c_{r}=c_{r}(t)$ such that the pressure admits the following decomposition 
$(2.3)$ :

$$
\begin{aligned}
p-c_{r}=- & \frac{|v|^{2}}{3}+p_{\text {loc }}+p_{\text {nonloc }} \\
:=- & \frac{|v|^{2}}{3}+\text { p.v. } \int_{B_{3 r}} K(x-y)(v \otimes v)(y) d y \\
& +\int_{\mathbb{R}^{3} \backslash B_{3 r}}(K(x-y)-K(-y))(v \otimes v)(y) d y
\end{aligned}
$$

in $L^{3 / 2}\left(B_{2 r}(0) \times(0, T)\right)$. By considering (2.3) in a larger region $B_{2} \times(0, T)$, we may remove the $r$-dependence of $c_{r}(t)$ by choosing $c_{r}(t)=c_{1}(t)$. Since $\left(v, p-c_{r}\right)$ is a suitable weak solution to (NS) in $B_{2 r}$, the local energy inequality (2.1) with the test function $\phi$ satisfying $\phi \in C_{0}^{\infty}\left(B_{2 r}\right)$ such that $0 \leq \phi \leq 1$ in $B_{2 r}$ with $\phi=1$ in $B_{r}$ and $\left\|\nabla^{k} \phi\right\|_{L^{\infty}} \leq C_{k} r^{-k}$ readily yields

$$
E_{r}(t) \leq \frac{2^{\alpha}}{(2 r)^{\alpha}} \int_{B_{2 r}}\left|v_{0}\right|^{2}+\frac{C}{r^{\alpha+2}} \int_{0}^{t} \int_{B_{2 r}}|v|^{2}+\frac{C}{r^{\alpha+1}} \int_{0}^{t} \int_{B_{2 r}}|v|^{3}+|v|\left|p-c_{r}\right| .
$$

By (3.6) together with Hölder and Young inequalities, the last term involving the pressure can be estimated as follows:

$$
\begin{aligned}
\frac{1}{r^{\alpha+1}} \int_{0}^{t} \int_{B_{2 r}}\left|v \| p-c_{r}\right| & \leq\left(\frac{1}{r^{\frac{3 \alpha}{2}}+\frac{1}{2}} \int_{0}^{t} \int_{B_{2 r}}|v|^{3}\right)^{\frac{1}{3}}\left(\frac{1}{r^{\frac{3 \alpha}{4}+\frac{5}{4}}} \int_{0}^{t} \int_{B_{2 r}}\left|p-c_{r}\right|^{\frac{3}{2}}\right)^{\frac{2}{3}} \\
& \leq \frac{1}{r^{\frac{3 \alpha}{2}+\frac{1}{2}}} \int_{0}^{t} \int_{B_{2 r}}|v|^{3}+\frac{C}{r^{\frac{3 \alpha}{4}+\frac{5}{4}}} \int_{0}^{t} \int_{B_{2 r}}\left|p-c_{r}\right|^{\frac{3}{2}} \\
& \leq \frac{1}{r^{\frac{3 \alpha}{2}+\frac{1}{2}}} \int_{0}^{t} \int_{B_{2 r}}|v|^{3}+\frac{C}{r^{\frac{3 \alpha}{4}+\frac{5}{4}}} \int_{0}^{t} \int_{B_{2 r}}|v|^{3}+\left|p_{l o c}\right|^{\frac{3}{2}}+\left|p_{\text {nonloc }}\right|^{\frac{3}{2}}
\end{aligned}
$$

Noting that $\frac{3 \alpha}{4}+\frac{5}{4} \leq \alpha+1 \leq \frac{3 \alpha}{2}+\frac{1}{2}$ for $\alpha \geq 1$, we have

$$
\begin{aligned}
E_{r}(t) & \leq 2^{\alpha} N_{R}+\frac{C}{r^{\alpha+2}} \int_{0}^{t} \int_{B_{2 r}}|v|^{2}+\frac{C}{r^{\frac{3 \alpha}{2}+\frac{1}{2}}} \int_{0}^{t} \int_{B_{2 r}}|v|^{3}+\left|p_{l o c}\right|^{\frac{3}{2}}+\frac{C}{r^{\frac{3 \alpha}{4}+\frac{5}{4}}} \int_{0}^{t} \int_{B_{2 r}}\left|p_{\text {nonloc }}\right|^{\frac{3}{2}} . \\
& =: 2^{\alpha} N_{R}+I_{\text {lin }}+I_{\text {nonlin }}+I_{\text {ploc }}+I_{\text {pnonloc. }}
\end{aligned}
$$

We divide the estimate into two cases.

Case I: $R<r \leq \frac{1}{6}$. This case is empty if $R>\frac{1}{6}$. For the simplicity of notation, let

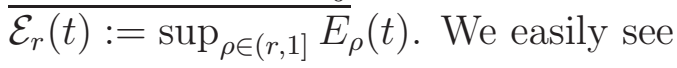

$$
I_{\text {lin }} \leq \frac{C}{R^{2}} \int_{0}^{t} \mathcal{E}_{R}(s) d s
$$


By the interpolation and Young's inequalities, we also have

$$
\begin{aligned}
I_{\text {nonlin }} & \leq \frac{C}{r^{\frac{3 \alpha}{2}+\frac{1}{2}}} \int_{0}^{t}\left(\int_{B_{r}}|\nabla v|^{2}\right)^{\frac{3}{4}}\left(\int_{B_{r}}|v|^{2}\right)^{\frac{3}{4}}+\left(\frac{1}{r} \int_{B_{r}}|v|^{2}\right)^{\frac{3}{2}} d s \\
& =\frac{C}{r^{2}} \int_{0}^{t}\left(\frac{1}{r^{\alpha-2}} \int_{B_{r}}|\nabla v|^{2}\right)^{\frac{3}{4}}\left(\frac{1}{r^{\alpha}} \int_{B_{r}}|v|^{2}\right)^{\frac{3}{4}}+\left(\frac{1}{r^{\alpha}} \int_{B_{r}}|v|^{2}\right)^{\frac{3}{2}} d s \\
& \leq \epsilon \mathcal{E}_{R}(t)+\frac{C_{\epsilon}}{R^{2}} \int_{0}^{t} \mathcal{E}_{R}(s)^{3}+\mathcal{E}_{R}(s)^{\frac{3}{2}} d s .
\end{aligned}
$$

This estimate and the Calderón-Zygmund estimate give

$$
\begin{aligned}
I_{p l o c} & \leq \frac{C}{r^{\frac{3 \alpha}{2}+\frac{1}{2}}} \int_{0}^{t} \int_{B_{3 r}}|v|^{3} \\
& \leq \epsilon \mathcal{E}_{R}(t)+\frac{C_{\epsilon}}{R^{2}} \int_{0}^{t} \mathcal{E}_{R}(s)^{3}+\mathcal{E}_{R}(s)^{\frac{3}{2}} d s .
\end{aligned}
$$

On the other hand, since $|x-y| \geq|y| / 3$ for $x \in B_{2 r}$ and $y \in \mathbb{R}^{3} \backslash B_{3 r}$, we see

$$
\begin{aligned}
\left|p_{\text {nonloc }}(x)\right| & \leq \int_{\mathbb{R}^{3} \backslash B_{3 r}}|K(x-y)-K(-y)||v(y)|^{2} d y \\
& \leq C r \int_{\mathbb{R}^{3} \backslash B_{3 r}} \frac{1}{|x-y|^{4}}|v(y)|^{2} d y \\
& \leq C r \int_{\mathbb{R}^{3} \backslash B_{3 r}} \frac{|v(y)|^{2}}{|y|^{4}} d y \\
& \leq C r \sum_{k=1}^{\left\lfloor-\log _{2} r-1\right\rfloor} \int_{B_{2^{k}} \backslash B_{2^{k-1}}} \frac{|v(y)|^{2}}{|y|^{4}} d y+C r \int_{\mathbb{R}^{3} \backslash B_{1 / 4}} \frac{|v(y)|^{2}}{|y|^{4}} d y \\
& \leq C r^{\alpha-3} \sum_{k=1}^{\left\lfloor-\log _{2} r-1\right\rfloor} \frac{1}{2^{(4-\alpha) k}}\left(\frac{1}{\left(2^{k} r\right)^{\alpha}} \int_{B_{2^{k}}}|v(y)|^{2} d y\right)+C r E_{\mathrm{uloc}} \\
& \leq C r^{\alpha-3} \mathcal{E}_{R}+C r\left\|v_{0}\right\|_{L_{\mathrm{uloc}}^{2}}^{2}
\end{aligned}
$$

provided $t \leq T_{\text {uloc }}=\min \left(T, \frac{c_{0}}{1+\left\|v_{0}\right\|_{L_{\text {uloc }}^{2}}^{4}}\right)$ and $\alpha<4$, where we have used Lemma 2.2 in the last line. We then obtain

$$
\begin{aligned}
I_{\text {pnonloc }} & \leq \frac{C}{r^{\frac{3 \alpha}{4}+\frac{5}{4}}} \int_{0}^{t} r^{3}\left(r^{\frac{3}{2}(\alpha-3)} \mathcal{E}_{R}^{\frac{3}{2}}(s)+r^{\frac{3}{2}}\left\|v_{0}\right\|_{L_{\text {uloc }}^{2}}^{3}\right) d s \\
& \leq \frac{C}{r^{2}} \int_{0}^{t} \mathcal{E}_{R}(s)^{\frac{3}{2}} d s+C t r^{\frac{13}{4}-\frac{3 \alpha}{4}}\left\|v_{0}\right\|_{L_{\text {uloc }}^{2}}^{3} \\
& \leq \frac{C}{r^{2}} \int_{0}^{t} \mathcal{E}_{R}(s)^{\frac{3}{2}} d s+\frac{\delta}{10}
\end{aligned}
$$

provided $t \leq\left(\frac{c \delta}{\left\|v_{0}\right\|_{L_{\text {uloc }}^{2}}^{3}}, T_{\text {uloc }}\right)$ with a small absolute constant $c>0$. We have used $\alpha \in[1,4)$ in the second inequality. Hence plugging (3.9), (3.10), (3.11), and (3.13) 
into (3.8) with $\epsilon=1 / 6$, we obtain

$$
\sup _{R<r<1 / 6} E_{r}(t) \leq \frac{\delta}{3}+\frac{1}{3} \mathcal{E}_{R}(t)+\frac{C}{R^{2}} \int_{0}^{t} \mathcal{E}_{R}(s)+\mathcal{E}_{R}(s)^{3} d s
$$

for $\delta \geq 70 N_{R}$.

Case II: $\frac{1}{6} \leq r \leq 1$. In order to bound the right hand side of (3.8), we observe from Lemma 2.2 that

$$
\sup _{1 / 3 \leq r \leq 2} E_{r}(t) \leq C E_{\text {uloc }}(t) \leq C\left\|v_{0}\right\|_{L_{\text {uloc }}^{2}}^{2} \quad \text { holds for } t \leq T_{\text {uloc }} .
$$

This shows

$$
I_{\text {lin }} \leq C \int_{0}^{t} \sup _{1 / 3 \leq r \leq 2} E_{r}(s) d s \leq C t\left\|v_{0}\right\|_{L_{\text {uloc }}^{2}}^{2}
$$

and hence if $t \leq\left(T_{\mathrm{uloc}}, \frac{c \delta}{\left\|v_{0}\right\|_{L_{\mathrm{uloc}}^{2}}^{2}}\right)$ with a suitable small constant $c>0$, we have

$$
I_{\text {lin }} \leq \frac{\delta}{40}
$$

For the nonlinear term in (3.8), we have

$$
\begin{aligned}
I_{\text {nonlin }} & \leq C \int_{0}^{t} \int_{B_{2}}|v|^{3} \\
& \leq C\left(\int_{0}^{t} \int_{B_{2}}|\nabla v|^{2}\right)^{\frac{3}{4}}\left(\int_{0}^{t}\left(\int_{B_{2}}|v|^{2}\right)^{3}\right)^{\frac{1}{4}}+C \int_{0}^{t}\left(\int_{B_{2}}|v|^{2}\right)^{\frac{3}{2}} \\
& \leq C\left(t^{\frac{1}{4}}+t\right)\left\|v_{0}\right\|_{L_{\text {uloc }}^{2}}^{3}, .
\end{aligned}
$$

By the Calderón-Zygmund estimate it also implies

$$
I_{p l o c} \leq C \int_{0}^{t} \int_{B_{3}}|v|^{3} \leq C\left(t^{\frac{1}{4}}+t\right)\left\|v_{0}\right\|_{L_{\text {uloc }}^{2}}^{3} .
$$

Thus the right hand sides in (3.16) and (3.17) are bounded by $\frac{\delta}{40}$, provided

$$
t \leq \min \left(T_{\mathrm{uloc}}, \frac{c \delta^{4}}{\left\|v_{0}\right\|_{L_{\mathrm{uloc}}^{2}}^{12}}, \frac{c \delta}{\left\|v_{0}\right\|_{L_{\mathrm{uloc}}^{2}}^{3}}\right)
$$

with some small absolute constant $c>0$. On the other hand, in the same way as in (3.12), we have

$$
\left|p_{\text {nonloc }}(x)\right| \leq C \int_{\mathbb{R}^{3} \backslash B_{1 / 2}} \frac{|v(y)|^{2}}{|y|^{4}} d y \leq C\left\|v_{0}\right\|_{L_{\text {uloc }}^{2}}^{2},
$$

which implies

$$
I_{\text {pnonloc }} \leq \frac{\delta}{40} \quad \text { for } t \leq \frac{c \delta}{\left\|v_{0}\right\|_{L_{\text {uloc }}^{2}}^{3}}
$$


Making use of these estimates in (3.8), we obtain

$$
\sup _{1 / 6 \leq r \leq 1} E_{r}(t) \leq \frac{\delta}{3}
$$

By choosing $c_{1}>0$ sufficiently small, we may take

$$
S_{1}:=\min \left(T, \frac{c_{1} \min \left\{1, \delta^{4}\right\}}{1+\left\|v_{0}\right\|_{L_{\text {uloc }}^{2}}^{12}}\right) \leq \min \left(T_{\text {uloc }}, \frac{c \delta}{\left\|v_{0}\right\|_{L_{\text {uloc }}^{2}}^{2}}, \frac{c \delta^{4}}{\left\|v_{0}\right\|_{L_{\text {uloc }}^{2}}^{12}}, \frac{c \delta}{\left\|v_{0}\right\|_{L_{\text {uloc }}^{2}}^{3}}\right) .
$$

Summarizing the conclusions of the cases I and II, we have

$$
\mathcal{E}_{R}(t) \leq \frac{\delta}{2}+\frac{C}{R^{2}} \int_{0}^{t} \mathcal{E}_{R}(s)+\mathcal{E}_{R}(s)^{3} d s
$$

for $t \leq S_{1}$. Applying Lemma 2.4 we obtain

$$
\mathcal{E}_{R}(t) \leq \delta \quad \text { for } t \leq \min \left(\lambda R^{2}, S_{1}\right), \quad \lambda=\frac{c}{1+\delta^{2}}
$$

Since $\delta \geq 70 N_{R} \geq 70 N_{r}$ for any $r \in(R, 1]$, we may replace $R$ by $r$ in (3.18), and thus we have verified (3.4) for $R<r \leq 1$.

To prove (3.5), we see that if $\lambda r^{2} \in\left(0, S_{1}\right]$,

$$
\begin{aligned}
& \frac{1}{r^{\frac{3 \alpha}{2}+\frac{1}{2}}} \int_{0}^{\lambda r^{2}} \int_{B_{r}}|v|^{3} \\
& \leq \frac{C}{r^{\frac{3 \alpha}{2}+\frac{1}{2}}} \int_{0}^{\lambda r^{2}}\left(\int_{B_{r}}|\nabla v|^{2}\right)^{\frac{3}{4}}\left(\int_{B_{r}}|v|^{2}\right)^{\frac{3}{4}} d s+\frac{C}{r^{\frac{3 \alpha}{2}+\frac{1}{2}}} \int_{0}^{\lambda r^{2}}\left(\frac{1}{r} \int_{B_{r}}|v|^{2}\right)^{\frac{3}{2}} d s \\
& \leq \frac{C}{r^{\frac{3 \alpha}{2}+\frac{1}{2}}} \int_{0}^{\lambda r^{2}}\left(\int_{B_{r}}|\nabla v|^{2}\right)^{\frac{3}{4}} d s r^{\frac{3 \alpha}{4}} E_{r}\left(\lambda r^{2}\right)^{\frac{3}{4}}+\frac{C}{r^{\frac{3 \alpha}{2}+\frac{1}{2}}} \int_{0}^{\lambda r^{2}} r^{\frac{3}{2}(\alpha-1)}\left(\frac{1}{r^{\alpha}} \int_{B_{r}}|v|^{2}\right)^{\frac{3}{2}} d s \\
& \leq \frac{C}{r^{\frac{3 \alpha}{2}+\frac{1}{2}}}\left(\int_{0}^{\lambda r^{2}} \int_{B_{r}}|\nabla v|^{2} d s\right)^{\frac{3}{4}}\left(\lambda r^{2}\right)^{\frac{1}{4}} r^{\frac{3 \alpha}{4}} E_{r}\left(\lambda r^{2}\right)^{\frac{3}{4}}+C \lambda E_{r}\left(\lambda r^{2}\right)^{\frac{3}{2}} \\
& \leq C\left(\lambda^{\frac{1}{4}}+\lambda\right) E_{r}\left(\lambda r^{2}\right)^{\frac{3}{2}} .
\end{aligned}
$$

Hence taking $R_{1}:=\min \left(\sqrt{\frac{S_{1}}{\lambda}}, 1\right)$,we have

$$
\frac{1}{r^{\frac{3 \alpha}{2}+\frac{1}{2}}} \int_{0}^{\lambda r^{2}} \int_{B_{r}}|v|^{3} \leq C \lambda^{\frac{1}{4}} \delta^{\frac{3}{2}} \quad \text { for } r \in\left(R, R_{1}\right] .
$$

By the Calderón-Zygmund estimate we also have

$$
\frac{1}{r^{\frac{3 \alpha}{2}+\frac{1}{2}}} \int_{0}^{\lambda r^{2}} \int_{B_{r}}\left|p_{l o c}\right|^{\frac{3}{2}} \leq C \lambda^{\frac{1}{4}} \delta^{\frac{3}{2}} \quad \text { for } r \in\left(R, R_{1}\right] .
$$

From (3.12) we have

$$
\begin{aligned}
\frac{C}{r^{\frac{3}{2} \alpha+\frac{1}{2}}} \int_{0}^{\lambda r^{2}} \int_{B_{2 r}}\left|p_{\text {nonloc }}\right|^{\frac{3}{2}} & \leq \frac{C}{r^{\frac{3}{2} \alpha+\frac{1}{2}}} \int_{0}^{\lambda r^{2}} r^{3}\left(r^{\frac{3}{2}(\alpha-3)} \mathcal{E}_{R}(s)^{\frac{3}{2}}+r^{\frac{3}{2}}\left\|v_{0}\right\|_{L_{\text {uloc }}^{2}}^{3}\right) d s \\
& \leq C \lambda\left(\delta^{\frac{3}{2}}+r^{6-\frac{3}{2} \alpha}\left\|v_{0}\right\|_{L_{\text {uloc }}^{2}}^{3}\right) .
\end{aligned}
$$


Combining this with (3.19) and (3.20) we obtain (3.5).

Proof of Theorem 3.1. By Lemma 2.5 using $R \leq 1 / 2$, for each $\delta \geq 2^{\alpha} N_{R}$ and $x_{0} \in$ $B_{1 / 2}$

$\sup _{R\left(x_{0}\right)<r \leq \rho} \frac{1}{r^{\alpha}} \int_{B_{r}\left(x_{0}\right)}\left|v_{0}\right|^{2} d x \leq \delta, \quad \rho=1-\left|x_{0}\right|, \quad R\left(x_{0}\right)=\max \left(\frac{R}{2}, 2\left(\frac{N_{R}}{\delta}\right)^{\frac{1}{\alpha}}\left|x_{0}\right|\right)$.

Let $v_{x_{0}}(x, t)=\rho v\left(x_{0}+\rho x, \rho^{2} t\right), p_{x_{0}}(x, t)=\rho^{2} p\left(x_{0}+\rho x, \rho^{2} t\right)$. Since $\left(v_{x_{0}}, p_{x_{0}}\right)$ solves (Ns) in $\mathbb{R}^{3} \times\left(0, \rho^{-2} T\right)$, corresponding to $(v, p)$ in $\mathbb{R}^{3} \times(0, T)$, and $1 / 2 \leq \rho \leq 1$, we have

$$
\begin{gathered}
\left\|v_{x_{0}}(t=0)\right\|_{L_{\text {uloc }}^{2}} \leq C\left\|v_{0}\right\|_{L_{\text {uloc }}^{2},} \\
N_{\rho^{-1} R\left(x_{0}\right)}\left(v_{x_{0}}\right)=\sup _{\rho^{-1} R\left(x_{0}\right)<r \leq 1} \frac{1}{r^{\alpha}} \int_{B_{r}(0)}\left|v_{x_{0}}(t=0)\right|^{2}=\sup _{R\left(x_{0}\right)<r \leq \rho} \frac{\rho^{\alpha-1}}{r^{\alpha}} \int_{B_{r}\left(x_{0}\right)}\left|v_{0}\right|^{2} \leq \delta .
\end{gathered}
$$

This ensures the assumption of Lemma 3.2 for $v_{x_{0}}$. If we take $\delta_{x_{0}}=70 \delta$, then from (3.5), there exists $c_{\left(x_{0}\right)}^{\prime}(t)$ such that

$$
\sup _{\rho^{-1} R\left(x_{0}\right)<r \leq R_{1}^{\prime}} \frac{1}{r^{2}} \int_{0}^{\lambda r^{2}} \int_{B_{r}(0)}\left|v_{x_{0}}\right|^{3}+\left|p_{x_{0}}-c_{\left(x_{0}\right)}^{\prime}(t)\right|^{\frac{3}{2}} \leq C\left(\lambda^{\frac{1}{4}} \delta^{\frac{3}{2}} r^{\frac{3}{2}(\alpha-1)}+\lambda\left\|v_{0}\right\|_{L_{\text {uloc }}^{2}}^{3} r^{\frac{9}{2}}\right) .
$$

Here

$$
R_{1}^{\prime}=\min \left(\sqrt{\frac{S_{1}^{\prime}}{\lambda}}, 1\right) \quad \text { with } \quad S_{1}^{\prime}=\min \left(\rho^{-2} T, \frac{c_{1} \min \left\{1, \delta^{4}\right\}}{1+\left\|v_{0}\right\|_{L_{\mathrm{uloc}}^{2}}^{12}}\right) .
$$

This implies

$$
\sup _{R\left(x_{0}\right) \leq r \leq \rho R_{1}^{\prime}} \frac{1}{\lambda r^{2}} \int_{0}^{\lambda r^{2}} \int_{B_{\sqrt{\lambda r}}\left(x_{0}\right)}|v|^{3}+\left|p-c_{\left(x_{0}\right)}(t)\right|^{\frac{3}{2}} \leq C\left(\lambda^{-\frac{3}{4}} \delta^{\frac{3}{2}} r^{\frac{3}{2}(\alpha-1)}+\left\|v_{0}\right\|_{L_{\text {uloc }}^{2}}^{3} r^{\frac{9}{2}}\right) .
$$

Denote

$$
\epsilon\left(x_{0}, r\right):=\frac{1}{r^{2}} \int_{0}^{r^{2}} \int_{B_{r}\left(x_{0}\right)}|v|^{3}+\left|p-c_{\left(x_{0}\right)}(t)\right|^{\frac{3}{2}} .
$$

For $\sqrt{\lambda} R\left(x_{0}\right) \leq r \leq \sqrt{\lambda} \rho R_{1}^{\prime}$ we have

$$
\begin{aligned}
\epsilon\left(x_{0}, r\right) & \leq C\left[\lambda^{-\frac{3}{4}} \delta^{\frac{3}{2}}\left(\lambda^{-1 / 2} r\right)^{\frac{3}{2}(\alpha-1)}+\left\|v_{0}\right\|_{L_{\text {uloc }}^{2}}^{3}\left(\lambda^{-1 / 2} r\right)^{\frac{9}{2}}\right] \\
& \leq C\left(1+\delta^{\frac{3 \alpha}{2}}\right) \delta^{\frac{3}{2}} r^{\frac{3}{2}(\alpha-1)}+C(1+\delta)^{\frac{9}{2}} r^{\frac{9}{2}}\left\|v_{0}\right\|_{L_{\text {uloc }}^{2}}^{3}
\end{aligned}
$$

We now choose $r>0$ as

$$
r \leq R_{2}:=\min \left(\left(\frac{c}{\left(1+\delta^{\alpha}\right) \delta}\right)^{\frac{1}{\alpha-1}}, \frac{c}{\left\|v_{0}\right\|_{L_{\text {uloc }}^{2}}^{2 / 3}(1+\delta)}\right)
$$

with a small constant $c>0$ so that $\epsilon\left(x_{0}, r\right)$ is smaller than $\epsilon_{\mathrm{CKN}}$ in Lemma 2.3. This enables us to apply Lemma 2.3 for $x_{0} \in B_{1 / 2}$ and

$$
t=r^{2} \in\left(\lambda R\left(x_{0}\right)^{2}, \min \left(\lambda\left(\rho R_{1}^{\prime}\right)^{2}, R_{2}^{2}\right)\right]
$$


to see that $v$ is regular at $\left(x_{0}, t\right)$ and, by $(3.22)$,

$$
\begin{aligned}
\left|v\left(x_{0}, t\right)\right| & \leq C_{\mathrm{CKN}} \epsilon\left(x_{0}, t^{\frac{1}{2}}\right)^{\frac{1}{3}} t^{-\frac{1}{2}} \\
& \leq C\left(1+\delta^{\frac{\alpha}{2}}\right) \delta^{\frac{1}{2}} t^{\frac{1}{4}(\alpha-3)}+C(1+\delta)^{\frac{3}{2}}\left\|v_{0}\right\|_{L_{\mathrm{uloc}}^{2}} t^{\frac{1}{4}} \\
& =C\left[\left(1+\delta^{\frac{\alpha}{2}}\right) \delta^{\frac{1}{2}}+(1+\delta)^{\frac{3}{2}}\left\|v_{0}\right\|_{L_{\mathrm{uloc}}^{2}} t^{\frac{1}{4}(4-\alpha)}\right] t^{\frac{1}{4}(\alpha-3)} .
\end{aligned}
$$

Since $1 / 2 \leq \rho \leq 1$, we may take $c>0$ so small that

$$
\begin{aligned}
\lambda\left(\rho R_{1}^{\prime}\right)^{2}=\rho^{2} \min \left(S_{1}^{\prime}, \lambda\right) & =\rho^{2} \min \left(\rho^{-2} T, \frac{c \min \left\{1, \delta^{4}\right\}}{1+\left\|v_{0}\right\|_{L_{\text {uloc }}^{2}}^{12}}, \lambda\right) \\
& \geq \min \left(T, \frac{c \min \left\{1, \delta^{4}\right\}}{1+\left\|v_{0}\right\|_{L_{\text {uloc }}^{2}}^{12}}, \frac{c}{(1+\delta)^{2}}\right) .
\end{aligned}
$$

Thus (3.23) is valid for $x_{0} \in B_{1 / 2}$ and $S_{a} \leq t \leq S_{b}$, where

$$
\begin{aligned}
S_{a} & :=\frac{c}{1+\delta^{2}} \max \left(R^{2}, C\left(N_{R} / \delta\right)^{\frac{2}{\alpha}}\left|x_{0}\right|^{2}\right), \\
S_{b} & :=\min \left(T, \frac{c \min \left\{1, \delta^{4}\right\}}{1+\left\|v_{0}\right\|_{L_{\mathrm{uloc}}^{2}}^{12}},\left(\frac{c}{\left(1+\delta^{\alpha}\right) \delta}\right)^{\frac{2}{\alpha-1}}, \frac{c}{\left(1+\left\|v_{0}\right\|_{L_{\mathrm{uloc}}^{2}}^{4 / 3}\right)(1+\delta)^{2}}\right) .
\end{aligned}
$$

If $N_{R} \geq 1$, we can take $\delta=70 N_{R}$. Then

$$
\begin{gathered}
S_{a} \leq \frac{c}{N_{R}^{2}} \max \left(R^{2},\left|x_{0}\right|^{2}\right), \\
S_{b} \geq S_{2}=\min \left(T, \frac{c}{1+\left\|v_{0}\right\|_{L_{\mathrm{uloc}}^{2}}^{12}},\left(\frac{c}{N_{R}}\right)^{\frac{2 \alpha+2}{\alpha-1}}, \frac{c}{\left(1+\left\|v_{0}\right\|_{L_{\mathrm{uloc}}^{2}}^{4 / 3}\right) N_{R}^{2}}\right),
\end{gathered}
$$

and

$$
\begin{array}{r}
\left|v\left(x_{0}, t\right)\right| \leq C\left[N_{R}^{(\alpha+1) / 2}+N_{R}^{3 / 2}\left\|v_{0}\right\|_{L_{\text {uloc }}^{2}} S_{2}^{\frac{1}{4}(4-\alpha)}\right] t^{\frac{1}{4}(\alpha-3)} \\
\text { for } \frac{c}{N_{R}^{2}} \max \left(R^{2},\left|x_{0}\right|^{2}\right) \leq t \leq S_{2} .
\end{array}
$$

If $N_{R} \leq 1$, we can take $\delta=70$. Then

$$
\begin{gathered}
S_{a} \leq c \max \left(R^{2}, N_{R}^{2 / \alpha}\left|x_{0}\right|^{2}\right), \\
S_{b} \geq S_{1}=\min \left(T, \frac{c}{1+\left\|v_{0}\right\|_{L_{\mathrm{uloc}}^{2}}^{12}}\right),
\end{gathered}
$$

(This $S_{1}$ may have a smaller $c$ than $S_{1}$ in Part (i)), and

$$
\begin{array}{r}
\left|v\left(x_{0}, t\right)\right| \leq C\left[1+\left\|v_{0}\right\|_{L_{\mathrm{uloc}}^{2}} S_{1}^{\frac{1}{4}(4-\alpha)}\right] t^{\frac{1}{4}(\alpha-3)} \\
\text { for } \quad c \max \left(R^{2}, N_{R}^{2 / \alpha}\left|x_{0}\right|^{2}\right) \leq t \leq S_{1} .
\end{array}
$$


Proof of Theorem 1.1.

For the proof of (1.3), we first claim that there exist $\epsilon_{*}$ and $c>0$ such that if $N_{0}:=N_{(\alpha), 0}:=\sup _{r \in(0,1]} \frac{1}{r^{\alpha}} \int_{B_{r}}\left|v_{0}(x)\right|^{2} d x<\epsilon_{*}$, then for any $x_{0} \in B_{\frac{1}{2}}$ and $r \in$ $\left(c N_{0}\left|x_{0}\right|^{\alpha}, c\left(1-\left|x_{0}\right|\right) R_{1}^{\prime}\right]$,

$$
\frac{1}{r^{2}} \int_{0}^{r^{2}} \int_{B_{r}\left(x_{0}\right)}|v|^{3}+\left|p-c_{\left(x_{0}\right)}(t)\right|^{\frac{3}{2}} d x d t \leq \epsilon_{\mathrm{CKN}}
$$

holds for some $c_{\left(x_{0}\right)}(t) \in \mathbb{R}$ and $R_{1}^{\prime}>0$ defined below. Here $\epsilon_{\mathrm{CKN}}$ is the small constant in Lemma 2.3. We observe from Lemma 2.5 (ii) that for each $\eta \geq 2 N_{0}$ and $x_{0} \in B_{\frac{1}{2}}$

$$
\sup _{R\left(x_{0}\right)<r \leq \rho} \frac{1}{r} \int_{B_{r}\left(x_{0}\right)}\left|v_{0}\right|^{2} d x \leq \eta, \quad \rho=1-\left|x_{0}\right|, \quad R\left(x_{0}\right)=\frac{2^{\alpha} N_{0}}{\eta}\left|x_{0}\right|^{\alpha} .
$$

Let $v_{x_{0}}(x, t)=\rho v\left(x_{0}+\rho x, \rho^{2} t\right), p_{x_{0}}(x, t)=\rho^{2} p\left(x_{0}+\rho x, \rho^{2} t\right)$ and $\delta=70 \eta$. As in the proof of Theorem 3.1 we have

$$
\begin{gathered}
\left\|v_{x_{0}}(t=0)\right\|_{L_{\mathrm{uloc}}^{2}} \leq C\left\|v_{0}\right\|_{L_{\text {uloc }}^{2}}, \\
\sup _{\rho^{-1} R\left(x_{0}\right)<r \leq 1} \frac{1}{r} \int_{B_{r}(0)}\left|v_{x_{0}}(t=0)\right|^{2}=\sup _{R\left(x_{0}\right)<r \leq \rho} \frac{1}{r} \int_{B_{r}\left(x_{0}\right)}\left|v_{0}\right|^{2} \leq \eta=\frac{\delta}{70} .
\end{gathered}
$$

This guarantees the assumption of Lemma 3.2 for $v_{x_{0}}$ and $\alpha=1$, and hence there exists $c_{\left(x_{0}\right)}^{\prime}(t)$ such that

$$
\sup _{\rho^{-1} R\left(x_{0}\right)<r \leq R_{1}^{\prime}} \frac{1}{r^{2}} \int_{0}^{\lambda r^{2}} \int_{B_{r}(0)}\left|v_{x_{0}}\right|^{3}+\left|p_{x_{0}}-c_{\left(x_{0}\right)}^{\prime}(t)\right|^{\frac{3}{2}} \leq C \lambda^{\frac{1}{4}} \delta^{\frac{3}{2}} .
$$

Here

$$
R_{1}^{\prime}=\min \left(\sqrt{\frac{S_{1}^{\prime}}{\lambda}}, 1\right) \quad \text { with } \quad S_{1}^{\prime}=\min \left(\rho^{-2} T, \frac{c_{1} \min \left\{1, \delta^{4}\right\}}{1+\left\|v_{0}\right\|_{L_{\mathrm{uloc}}^{2}}^{12}}\right) .
$$

This implies

$$
\sup _{R\left(x_{0}\right) \leq r \leq \rho R_{1}^{\prime}} \frac{1}{\lambda r^{2}} \int_{0}^{\lambda r^{2}} \int_{B_{\sqrt{\lambda} r}\left(x_{0}\right)}|v|^{3}+\left|p-c_{\left(x_{0}\right)}(t)\right|^{\frac{3}{2}} \leq \frac{C \delta^{\frac{3}{2}}}{\lambda^{\frac{3}{4}}} \leq C\left(1+\delta^{\frac{3}{2}}\right) \delta^{\frac{3}{2}} .
$$

Take a constant $\delta_{0}>0$ so small that $C\left(1+\delta_{0}^{\frac{3}{2}}\right) \delta_{0}^{\frac{3}{2}} \leq \epsilon_{\mathrm{CKN}}$ and assume that $v_{0}$ satisfies $N_{0} \leq \delta_{0} / 70$. Then we may choose $\delta=\delta_{0}$ since $\delta_{0} \geq 70 N_{0}$. With this choice and with $\lambda_{0}=\lambda\left(\delta_{0}\right)$, we have verified (3.27) for $\lambda_{0}^{\frac{1}{2}} R\left(x_{0}\right)<r \leq \lambda_{0}^{\frac{1}{2}} \rho R_{1}^{\prime}$.

This enables us to apply Lemma 2.3 for $x_{0} \in B_{1 / 2}$ and $t_{0}=r^{2} \in\left(C \lambda_{0}\left(\frac{N_{0}}{\delta_{0}}\right)^{2}\left|x_{0}\right|^{2 \alpha}, \lambda_{0}\left(\rho R_{1}^{\prime}\right)^{2}\right]$ to see

$$
\left|v\left(x_{0}, t_{0}\right)\right| \leq C_{\mathrm{CKN}} r^{-1}=C_{\mathrm{CKN}} t_{0}^{-\frac{1}{2}}
$$


and hence $v$ is regular at $\left(x_{0}, t_{0}\right)$. Since $\lambda_{0}$ and $\delta_{0}$ are absolute constants and $1 / 2 \leq$ $\rho \leq 1$, we may choose $c>0$ so small that

$$
\begin{aligned}
\lambda_{0}\left(\rho R_{1}^{\prime}\right)^{2}=\rho^{2} \min \left(S_{1}^{\prime}, \lambda_{0}\right) & =\rho^{2} \min \left(\rho^{-2} T, \frac{c \min \left\{1, \delta_{0}^{4}\right\}}{1+\left\|v_{0}\right\|_{L_{\mathrm{uloc}}^{2}}^{12}}, \lambda_{0}\right) \\
& \geq \min \left(T, \frac{c}{1+\left\|v_{0}\right\|_{L_{\mathrm{uloc}}^{2}}^{12}}\right) .
\end{aligned}
$$

Thus $\left|v\left(x_{0}, t_{0}\right)\right| \leq C_{\mathrm{CKN}} t_{0}^{-\frac{1}{2}}$ for $x_{0} \in B_{1 / 2}$ and $c N_{0}^{2}\left|x_{0}\right|^{2 \alpha} \leq t_{0} \leq \min \left(T, \frac{c}{1+\left\|v_{0}\right\|_{L_{\text {uloc }}^{2}}^{12}}\right)$. This proves (1.3) for the case $N_{0} \leq \epsilon_{*}$.

In order to consider the case $N_{0}>\epsilon_{*}$, let $u(x, t)=\rho v\left(\rho x, \rho^{2} t\right), q(x, t)=\rho^{2} p\left(\rho x, \rho^{2} t\right)$, $u_{0}(x)=\rho v_{0}(\rho x)$ with $0<\rho<1$ to be given below. We easily see

$$
\left\|u_{0}\right\|_{L_{\text {uloc }}^{2}}=\rho^{-\frac{1}{2}} \sup _{x_{0} \in \mathbb{R}^{3}}\left\|v_{0}\right\|_{L^{2}\left(B_{\rho}\left(x_{0}\right)\right)} \leq C \rho^{-\frac{1}{2}}\left\|v_{0}\right\|_{L_{\text {uloc }}^{2}}
$$

$$
N_{0}\left(u_{0}\right)=\sup _{r \in(0,1]} \frac{1}{r^{\alpha}} \int_{B_{r}}\left|u_{0}\right|^{2}=\sup _{r \in(0,1]} \frac{1}{\rho r^{\alpha}} \int_{B_{\rho r}}\left|v_{0}\right|^{2}=\sup _{r \in(0, \rho]} \frac{\rho^{\alpha-1}}{r^{\alpha}} \int_{B_{r}}\left|v_{0}\right|^{2} \leq \rho^{\alpha-1} N_{0} .
$$

Let $\rho=\left(\frac{\epsilon_{*}}{N_{0}}\right)^{\frac{1}{\alpha-1}}$ so that $N_{0}\left(u_{0}\right) \leq \epsilon_{*}$. Then it follows from Step 1 that $u$ is regular and satisfies $|u(x, t)| \leq C_{\mathrm{CKN}} t^{-\frac{1}{2}}$ in $\Pi$ in the region

$$
\left\{(x, t) \in B_{\frac{1}{2}} \times\left(0, \rho^{-2} T\right) ; c N_{0}\left(u_{0}\right)^{2}|x|^{2 \alpha} \leq t \leq \frac{c}{1+\left\|u_{0}\right\|_{L_{\text {uloc }}^{2}}^{12}}\right\} .
$$

Rescaling back to $(v, p)$ and using (3.29), we see that $v$ is regular and satisfies $|v(x, t)| \leq C_{\mathrm{CKN}} t^{-\frac{1}{2}}$ in the region

$$
\left\{(x, t) \in B_{\frac{\rho}{2}} \times(0, T) ; c N_{0}^{2}|x|^{2 \alpha} \leq t \leq \frac{c \rho^{2}}{1+\rho^{-6}\left\|v_{0}\right\|_{L_{\mathrm{uloc}}^{2}}^{12}}\right\} .
$$

By taking $c>0$ sufficiently small, we see that this region contains

$$
\Pi_{1}=\left\{(x, t) \in B_{\frac{1}{2}} \times(0, T) ; c N_{0}^{2}|x|^{2 \alpha} \leq t \leq T_{1}\right\}, \quad T_{1}:=\frac{c}{N_{0}^{\frac{2}{\alpha-1}}\left(1+N_{0}^{\frac{6}{\alpha-1}}\left\|v_{0}\right\|_{L_{\text {uloc }}^{2}}^{12}\right)}
$$

This completes the proof of (1.3).

Est. (1.4) is the special case of Theorem 3.1 with $R=0$. Indeed for all $N_{0}<\infty$ we have

$$
|v(x, t)| \leq M t^{\frac{\alpha-3}{4}} \quad\left(C_{1}|x|^{2}<t<S_{2}\right),
$$

where $M=C\left[1+\left\|v_{0}\right\|_{L_{\text {uloc }}^{2}} S_{1}^{\frac{1}{4}(4-\alpha)}\right]$ if $N_{0} \leq 1$, and $M=C\left[N_{0}^{(\alpha+1) / 2}+N_{0}^{3 / 2}\left\|v_{0}\right\|_{L_{\text {uloc }}^{2}} S_{2}^{\frac{1}{4}(4-\alpha)}\right]$ if $N_{0} \geq 1$. Hence we can take

$$
C_{0}=C\left[1+N_{0}^{(\alpha+1) / 2}+\left(1+N_{0}^{3 / 2}\right)\left\|v_{0}\right\|_{L_{\text {uloc }}^{2}} S_{1}^{\frac{1}{4}(4-\alpha)}\right] .
$$


We also have $C_{1}=c N_{0}^{2 / \alpha}$ if $N_{0} \leq 1$, and $C_{1}=c N_{0}^{-2}$ if $N_{0} \geq 1$. Hence we can take

$$
C_{1}=\frac{c N_{0}^{2 / \alpha}}{\left(1+N_{0}\right)^{2+2 / \alpha}} .
$$

Therefore (3.31) implies (1.4) of Theorem 1.1. This completes the proof.

Proof of Theorem 1.2. The proof is similar to that of (1.4), but we give the details for completeness. We first observe that

$$
N\left(x_{0}\right):=\sup _{r \in(0,1]} \frac{1}{r^{\alpha}} \int_{B_{r}\left(x_{0}\right)}\left|v_{0}\right|^{2} \leq C\left\|v_{0}\right\|_{L^{q}}^{2}<\infty
$$

for $x_{0} \in B_{1}$ with $\alpha=3-\frac{6}{q} \in(1,3]$ for $3<q \leq \infty$. Here we write $\|\cdot\|_{L^{q}}=\|\cdot\|_{L^{q}\left(B_{2}\right)}$. For each $x_{0} \in B_{1}$ we may adapt the proof of (1.4) for $v\left(x+x_{0}, t\right)$ and $R=0$. Indeed for any $\delta \geq 70 N\left(x_{0}\right)$ restricting the time variable in (3.23) and (3.24) as $\widetilde{S}_{a} \leq t \leq \widetilde{S}_{b}$ with

$$
\begin{aligned}
& \widetilde{S}_{a}:=\frac{c}{1+\delta^{2}}\left(\frac{N\left(x_{0}\right)}{\delta}\right)^{\frac{2}{\alpha}}\left|x-x_{0}\right|^{2}, \\
& \widetilde{S}_{b}:=\min \left(S_{b}, \frac{1}{1+\delta^{2}}\left(\frac{c \delta}{\left\|v_{0}\right\|_{L_{\mathrm{uloc}}^{2}}}\right)^{\frac{4}{4-\alpha}}\right),
\end{aligned}
$$

we have

$$
|v(x, t)| \leq C\left[\left(1+\delta^{\frac{\alpha}{2}}\right) \delta^{\frac{1}{2}}+(1+\delta)^{\frac{3}{2}}\left\|v_{0}\right\|_{L_{\mathrm{uloc}}^{2}} t^{\frac{1}{4}(4-\alpha)}\right] t^{\frac{1}{4}(\alpha-3)} \leq C\left(1+\delta^{\frac{\alpha}{2}}\right) \delta^{\frac{1}{2}} t^{\frac{1}{4}(\alpha-3)} .
$$

The second inequality is by the second argument of $\widetilde{S}_{b}$. By (3.32), taking $C>0$ sufficiently big, we may choose $\delta=C\left\|v_{0}\right\|_{L^{q}}^{2}$ uniformly on $x_{0} \in B_{1}$. Taking $x=x_{0}$, this implies

$$
\|v(t)\|_{L^{\infty}\left(B_{1}\right)} \leq C\left(1+\left\|v_{0}\right\|_{L^{q}}^{\alpha}\right)\left\|v_{0}\right\|_{L^{q}} t^{\frac{1}{4}(\alpha-3)}
$$

for $0<t<T_{3}$ where

$$
\begin{aligned}
T_{3}=\min \left(T, \frac{c \min \left(1,\left\|v_{0}\right\|_{L^{q}}^{8}\right)}{1+\left\|v_{0}\right\|_{L_{\mathrm{uloc}}^{2}}^{2}},\left(\frac{c}{\left(1+\left\|v_{0}\right\|_{L^{q}}^{\alpha}\right)\left\|v_{0}\right\|_{L^{q}}}\right)^{\frac{4}{\alpha-1}}\right. \\
\\
\left.\frac{c}{\left(1+\left\|v_{0}\right\|_{L_{\mathrm{uloc}}^{2 / 3}}^{2 / 3}\right)\left(1+\left\|v_{0}\right\|_{L^{q}}^{4}\right)}, \frac{1}{1+\left\|v_{0}\right\|_{L^{q}}^{4}}\left(\frac{c\left\|v_{0}\right\|_{L^{q}}^{2}}{\left\|v_{0}\right\|_{L_{\mathrm{uloc}}^{2}}^{2}}\right)^{\frac{4}{4-\alpha}}\right) .
\end{aligned}
$$

This shows the desired claim.

Proof of Theorem 1.3. For $\alpha:=-\beta>1$ we observe that

$$
\frac{1}{r^{\alpha}} \int_{B_{r}}\left|v_{0}\right|^{2} \leq \int_{B_{r}}|x|^{\beta}\left|v_{0}\right|^{2} \leq L:=\left\|v_{0}\right\|_{L^{2, \beta}\left(\mathbb{R}^{3}\right)}^{2}<\infty .
$$


Hence $N_{(\alpha)}\left(v_{0}\right)=\sup _{r \in(0,1]} \frac{1}{r^{\alpha}} \int_{B_{r}}\left|v_{0}\right|^{2} \leq L$. By (1.3) of Theorem 1.1, $v$ is regular and satisfies $|v(x, t)| \leq C t^{-1 / 2}$ in the region

$$
\left\{(x, t) \in B_{\frac{1}{2}} \times(0, \infty): c_{1} L|x|^{2 \alpha} \leq t<T_{1}\right\}
$$

with $T_{1}=T_{1}\left(\left\|v_{0}\right\|_{L_{\text {uloc }}^{2}}, L, \alpha\right)$, while $c_{1}$ and $C$ depend only on $\alpha$. Let $T_{4}=\min \left(T_{1}, c_{1} L 2^{-2 \alpha}\right)$. The region

$$
\left\{(x, t) \in \mathbb{R}^{3} \times(0, \infty): c_{1} L|x|^{2 \alpha} \leq t<T_{4}\right\}
$$

is a subset of (3.35). This finishes the proof of Theorem 1.3.

\section{REFERENCES}

[1] Barker, T. and Prange, C., Localized smoothing for the Navier-Stokes equations and concentration of critical norms near singularities, Arch. Rational Math. Anal. 236 (2020), no. 3, 1487-1541.

[2] Barker, T. and Prange, C., Quantitative regularity for the Navier-Stokes equations via spatial concentration, arXiv preprint:2003.06717.

[3] Bradshaw, Z., Kukavica, I., and Tsai, T.-P., Existence of global weak solutions to the Navier-Stokes equations in weighted spaces, to appear in Indiana Univ. Math. J., arXiv preprint:1910.06929.

[4] Bradshaw, Z. and Tsai, T.-P., Global existence, regularity, and uniqueness of infinite energy solutions to the Navier-Stokes equations, Comm. Partial Differential Equations 45 (2020), no. 9, 1168-1201.

[5] Caffarelli, L., Kohn, R., and Nirenberg, L., Partial regularity of suitable weak solutions of the Navier-Stokes equations. Comm. Pure Appl. Math. 35 (1982), no. 6, 771-831.

[6] D'Ancona, P. and Lucá R., On the regularity set and angular integrability for the Navier-Stokes equation. Arch. Ration. Mech. Anal. 221 (2016), no. 3, 1255-1284.

[7] Fabes, E. B., Jones, B.F., and Rivière, N. M., The initial value problem for the Navier-Stokes equations with data in $L^{p}$, Arch. Rational Mech. Anal. 45 (1972), 222-240.

[8] Fernández-Dalgo, P. G. and Lemarié-Rieusset, P. G., Weak solutions for Navier-Stokes equations with initial data in weighted $L^{2}$ spaces, Arch. Ration. Mech. Anal. 237 (2020), no. 1, $347-382$.

[9] Giga, Y. and Miyakawa, T., Solutions in $L^{r}$ of the Navier-Stokes initial value problem. Arch. Rational Mech. Anal. 89 (1985), 267-281.

[10] Hopf, E., Über die Aufgangswertaufgave für die hydrodynamischen Grundliechungen. Math. Nachr. 4 (1951), 213-231.

[11] Jia, H. and Šverák, V., Minimal $L^{3}$-initial data for potential Navier-Stokes singularities. SIAM J. Math. Anal. 45 (2013), no. 3, 1448-1459.

[12] Jia, H. and Šverák, V., Local-in-space estimates near initial time for weak solutions of the Navier-Stokes equations and forward self-similar solutions. Invent. Math. 196 (2014), no. 1, 233-265.

[13] Jia, H., Šverák, V., and Tsai, T.-P., Self-similar solutions to the nonstationary Navier-Stokes equations, Handbook of Mathematical Analysis in Mechanics of Viscous Fluids, 461-507, Springer, 2018.

[14] Kang, K., Miura, H., and Tsai, T.-P., Short time regularity of Navier-Stokes flows with locally $L^{3}$ initial data and applications, Int. Math. Res. Not. rnz327 https://doi.org/10.1093/imrn/rnz327 arXiv preprint:1812.10509.

[15] Kang, K., Miura, H., and Tsai, T.-P., Regular sets and an $\epsilon$-regularity theorem in terms of initial data for the Navier-Stokes equations, Pure and Applied Analysis, to appear. arXiv preprint: 2006.13145. 
[16] Kato, T., Strong $L^{p}$-solutions of the Navier-Stokes equation in $R^{m}$, with applications to weak solutions, Math. Z., 187 (1984), pp. 471-480.

[17] Kikuchi, N. and Seregin, G., Weak solutions to the Cauchy problem for the Navier-Stokes equations satisfying the local energy inequality. Nonlinear equations and spectral theory, 141164, Amer. Math. Soc. Transl. Ser. 2, 220, Amer. Math. Soc., Providence, RI, 2007.

[18] Kwon, H. and Tsai, T.-P., Global Navier-Stokes flows for non-decaying initial data with slowly decaying oscillation, Comm. Math. Phys. 375, 1665-1715 (2020).

[19] Lemarié-Rieusset, P. G., Recent developments in the Navier-Stokes problem. Chapman Hall/CRC Research Notes in Mathematics, 431. Chapman Hall/CRC, Boca Raton, FL, 2002.

[20] Lemarié-Rieusset, P. G., The Navier-Stokes problem in the 21st century. CRC Press, Boca Raton, FL, 2016.

[21] Leray, J., Sur le mouvement d'un liquide visqueux emplissant l'espace. (French) Acta Math. 63 (1934), no. 1, 193-248.

[22] Lin, F., A new proof of the Caffarelli-Kohn-Nirenberg theorem, Comm. Pure Appl. Math. 51 (1998), no. 3, 241-257.

[23] Maekawa, Y., Miura, H., and Prange, C., Local energy weak solutions for the Navier-Stokes equations in the half-space, Comm. Math. Phys. 367 (2019), no. 2, 517-580.

[24] Nečas, J., Růžička, M., and Šverák, V., On Leray's self-similar solutions of the Navier-Stokes equations, Acta Math. 176 (1996), 283-294.

[25] Tao, T., Localisation and compactness properties of the Navier-Stokes global regularity problem. Anal. PDE 6 (2013), no. 1, 25-107.

(K. Kang) Department of Mathematics, Yonsei University, Seoul 120-749, South KOREA

Email address: kkang@yonsei.ac.kr

(H. Miura) Department of Mathematical and Computing Science, Tokyo Institute of Technology, Tokyo 152-8551, JAPAN

Email address: miura@is.titech.ac.jp

(T.-P. Tsai) Department of Mathematics, University of British Columbia, VancouVER, BC V6T 1Z2, CANADA

Email address: ttsai@math.ubc.ca 